Bond University

Research Repository

\title{
Which Patient Factors Best Predict Discharge Destination After Primary Total Knee Arthroplasty? The ARISE Trial
}

Sattler, Larissa N.; Hing, Wayne A.; Rathbone, Evelyne N.; Vertullo, Christopher J.

Published in:

Journal of Arthroplasty

DOI:

10.1016/j.arth.2020.05.056

Licence:

CC BY-NC-ND

Link to output in Bond University research repository.

Recommended citation(APA):

Sattler, L. N., Hing, W. A., Rathbone, E. N., \& Vertullo, C. J. (2020). Which Patient Factors Best Predict

Discharge Destination After Primary Total Knee Arthroplasty? The ARISE Trial. Journal of Arthroplasty, 35(10),

2852-2857. https://doi.org/10.1016/j.arth.2020.05.056

\section{General rights}

Copyright and moral rights for the publications made accessible in the public portal are retained by the authors and/or other copyright owners and it is a condition of accessing publications that users recognise and abide by the legal requirements associated with these rights.

For more information, or if you believe that this document breaches copyright, please contact the Bond University research repository coordinator. 
1 Which Patient Factors Best Predict Discharge Destination After Primary Total Knee

2 Arthroplasty? The ARISE Trial.

3 Abstract

$4 \quad$ Background

5 The role of inpatient rehabilitation after total knee arthroplasty (TKA) remains uncertain,

6 with evidence suggesting no better functional outcomes for those who discharge to

7 rehabilitation to those who discharge home. The aim of this study was to develop and

8 implement a preoperative predictive tool, ARISE (Arthroplasty Rehabilitation Initial

9 Screening Evaluation), that incorporated psychological, functional, and socio-demographic factors to determine discharge destination.

Methods

One week prior to TKA, the ARISE tool was administered to 100 patients, in addition to an EQ-5D-5L survey and other demographic data being recorded. The primary outcome was discharge destination. An enhanced recovery pathway, which included an anaesthetic protocol designed to optimise early mobilisation, was utilised. Univariable and multivariable logistic regression analysis was performed to determine the likelihood of discharge destination.

Results

Patients in the rehabilitation group were, on average, 4.5 years older than the home group $(\mathrm{P}=0.036)$. After multivariable regression, ARISE questions that were predictive of discharge destination related to beliefs around the superiority of inpatient rehabilitation $(\mathrm{OR}=9.9$ [2.637.9]) and post-discharge level of support ( $\mathrm{OR}=6.3$ [1.5-26.8]). No question around selfreported physical function was predictive.

\section{Conclusion}

Pre-operative patient beliefs regarding rehabilitation and future home support are highly predictive of discharge destination after primary TKA. Pre-operative patient reported functional status and demographic variables, with the exception of increasing age, were not shown to be predictive. Predicting those that are most likely to discharge to rehabilitation allows for early, targeted interventions to optimise resources and increase likelihood of home discharge. 
Total knee arthroplasty (TKA); Rehabilitation; Discharge Destination; Predictors

\section{Introduction}

Worldwide rates of total knee arthroplasty (TKA) are expected to continue to rise [1-4] on the background of an aging population and a global obesity epidemic [5-8]. The increasing prevalence and cost of TKA will have significant economic implications which will lead to greater emphasis on controlling expenditure without compromising patient outcomes [9].

Post-acute care comprises a significant portion of the costs associated with TKA, reportedly being over one third of total episode of care costs [10]. One of the most substantial post-acute care costs is discharge to inpatient rehabilitation, with those who do having a greater associated cost burden when compared to those TKA patients that discharge to home [9, 11]. With this increased demand for arthroplasty there will be a coincidental increase in the number of those discharging to inpatient rehabilitation after TKA. Currently, there is a paucity of evidence supporting discharge to inpatient rehabilitation over discharge home after uncomplicated, primary TKA, with the recommendation of home discharge being the primary aim in this population [11]. Moreover, large unexplained regional variations in discharge destination currently exist [12].

Predicting pre-operatively those patients with the greatest likelihood of discharging to inpatient rehabilitation allows for early and targeted interventions designed to increase the likelihood of safe discharge directly home. A tool which can effectively predict discharge destination, prior to surgery also allows for the optimisation of resource allocation. The preoperative organisation of home-based services or inpatient rehabilitation facility admission allows discharge planning to be initiated prior to surgery. Additionally, identifying those patients that have pre-operative concerns about returning directly to their home environment after TKA provides opportunity for discussion and reassurance regarding the objective measures the clinical team uses to assess readiness and safety for home discharge from the acute hospital setting.

Previous tools designed to predict discharge destination have failed to demonstrate a high level of accuracy in those TKA patients with a "medium" level of risk for a "non-home" discharge, which includes inpatient rehabilitation [13-16]. This issue is further heightened as the "medium" category is comprised of the largest number of patients, when compared to 
those considered as "low" or "high risk", for discharge to inpatient rehabilitation [13-16]. While, patient expectation of discharge destination has been shown to be the most predictive factor of actual discharge destination, this component has not been included in the scoring models of existing predictive tools [17]. Moreover, while the influence of patient expectation on discharge destination after TKA has been reported, the reasons behind that expectation has yet to be explored $[14,15,17]$.

The aim of this trial was to develop and administer a questionnaire comprised of sociodemographic, functional and psychological domains, the ARISE (Arthroplasty Rehabilitation Initial Screening Evaluation) tool, to identify which patient factors best predict discharge destination in a primary TKA population.

\section{Methods}

\section{Trial design}

This study was a prospective cohort trial, designed and reported in accordance with the Strengthening the Reporting of Observational Studies in Epidemiology (STROBE) statement guidelines [18]. Institutional review board approval was obtained, and the trial was prospectively registered at Australian New Zealand Clinical Trials Registry (Identifier ACTRN12619001483145).

\section{Questionnaire design and development}

The construction of the ARISE tool was based on the International Association for Medical Education (AMEE) guidelines for developing questionnaires, which is a systematic, sevenstep process for designing high-quality questionnaires [19]. A literature review of existing tools was performed to establish the first version of the ARISE tool question items and response categories were based on a Likert-style scale. Patient interviews were then conducted to make further refinements. Expert validation was conducted through interviews with independent orthopaedic specialists and physiotherapists before administering the fourth and final version of the ARISE tool.

\section{Participants}

Patients of a high volume, multi-surgeon, elective arthroplasty hospital scheduled to undergo unilateral primary TKA for a primary diagnosis of OA were eligible for inclusion. The only exclusion criteria was patients who had received a contralateral TKA in the past 12 months. 
Patients were enrolled one week prior to their scheduled TKA from November 2019 to January 2020 and demographic variables, including age, gender, body mass index and the American Society of Anaesthesiologist (ASA) score, were recorded. The ARISE tool was independently administered in the week prior to surgery, as well as an EQ-5D-5L [20]. The EQ-5D-5L is a validated survey for measuring health-related quality of life and it also includes a visual analogue scale for self-rated health. Post-operatively, the EQ-5D-5L was administered again on day of discharge from hospital. The length of stay, in number of days, in the acute hospital setting was recorded along with any complication resulting in return to theatre or hospital readmission. Discharge destination (home or inpatient rehabilitation) was the primary outcome of interest. A sample size of 100 patients was estimated based on previous published literature on developing pre-operative tools to predict outcomes after TKA $[21,22]$. The sample size was confirmed with a calculation using published TKR private hospital procedure rates and the proportion of which discharge to inpatient rehabilitation, using a confidence level of $95 \%$ and confidence limits of $10 \%$, a sample size of 92 was estimated.

\section{Perioperative protocols}

All patients received a cemented minimally stabilized total knee prosthesis with patella resurfacing. The anaesthetic protocol included spinal anaesthesia, an adductor canal nerve block, and a peri-articular/capsular injection of local anaesthetic to the operative limb. Postoperatively, patients underwent an enhanced recovery pathway which included early mobilisation and a three-exercise pedalling-based protocol which was supervised by a physiotherapist twice daily until discharge [23]. The criteria for home discharge was independent transfers and mobility with the walking aid to be used at home, stair climbing practice, and knee flexion range of motion to 90 degrees.

\section{Statistical analysis}

Data were analysed using the Statistical Package for Social Sciences (SPSS version 26). Descriptive statistics for continuous data are expressed as mean (SD) or median (range) depending on data distribution, and statistical significance considered as $P$ values $<0.05$. Categorical variables were summarised using counts and percentages. Differences in demographic variables between discharge groups were analysed by the chi-square test with respect to categorical data. Normally distributed continuous data were analysed using an independent samples $t$-test. The non-parametric Mann-Whitney U test was used when data 
were not normally distributed. The relationship between each predictive variable and discharge destination was assessed using the chi-square test or the Fisher's exact test. However, since some cells had a low response count, prior to the main analyses, the Likert responses were re-categorised to two levels. Univariable logistic regression analysis was initially performed to determine the likelihood of discharge destination for each variable. Predictor variables that were significant at the 0.1 were selected for potential inclusion in a multivariable analysis and underwent backward stepwise logistic regression to determine the model that best predicted inpatient rehabilitation discharge. A C-statistic was also generated to assess goodness-of-fit and predictive accuracy of the final logistic regression model.

\section{Results}

\section{Baseline characteristics and discharge destination}

In total, 100 participants from four arthroplasty surgeons were enrolled. All 100 participants completed the ARISE questionnaire and the patient characteristics are described in Table 1. Of the 100 participants, 82 discharged home and 18 discharged to inpatient rehabilitation. On average, patients in the rehabilitation group were 4.5 years older than those in the home group $(\mathrm{P}=0.036)$, and of those who were aged greater than 75 years, a greater proportion discharged to inpatient rehabilitation $(\mathrm{P}=0.030)$. No differences between groups were found in gender, body mass index, and comorbidity status.

\section{The ARISE tool}

The univariable results revealed 5 ARISE questions that were individually predictive of discharge destination, they are listed in Table 2. The predictive questions related to the patient's belief about their post-discharge level of support, their pre-operative beliefs regarding the superiority of inpatient rehabilitation or their self-assessed ability to perform self-directed home-based exercises. If a patient agreed or strongly agreed that they would do best with inpatient rehabilitation, they were 33 times more likely to discharge to inpatient rehabilitation $(\mathrm{OR}=32.8$ [8.0 to 129.9], $P<0.001)$ and if they were worried most or all of the time about being a burden on their family or friends, then inpatient rehabilitation discharge was 8 times more likely ( $\mathrm{OR}=8.1$ [2.6 to 24.9 ], $P<0.001$ ). A patient who did not have someone, all or most of the time, who could help them after surgery resulted in an 11 times greater likelihood of discharge to inpatient rehabilitation ( $\mathrm{OR}=10.7$ [3.2 to 35.8], $P<0.001$ ). No question around physical function or living situation was predictive of discharge destination. 
In finalising the multivariable stepwise regression, and to avoid multicollinearity, the 3 questions around a patient's beliefs about the superiority of rehabilitation were combined to a create new variable. The results show that if a patient agreed to at least 2 of those three statements about rehabilitation, then the patient was 10 times more likely ( $\mathrm{OR}=9.9$ [2.6 to 37.9]) to be discharged to rehabilitation. When this model was adjusted for age; a patient aged 75 years and over was more than three and a half times more likely for inpatient rehabilitation discharge $(\mathrm{OR}=3.6$ [0.9 to 13.6]). Not having someone to help after surgery made inpatient rehabilitation discharge six times more likely ( $\mathrm{OR}=6.3$ [1.5 to 26.8]) (Table 3). The final multivariable regression model produced a C-statistic of 0.84 , demonstrating a strong model with $84 \%$ correct predictivity.

EQ-5D-5L and Length of stay

Results of the EQ-5D-5L and length of stay are shown in Table 4. The self-rated global health score, as measured by the EQ-5D-5L VAS, was 10 points (on a 100-point scale) better for the home discharge group versus the inpatient rehabilitation discharge group, both preoperatively $(P=0.043)$ and on day of discharge $(P=0.009)$ from the acute hospital setting. However, the EQ-5D-5L total showed no significant difference for discharge destination $(P=$ 0.211). Length of stay for the rehabilitation group was one day longer, at 4 days, when compared to the home discharge group at 3 days $(P<0.001)$. There were no reported complications resulting in return to theatre or hospital readmission for either group.

\section{Discussion}

The most significant finding of this study was that discharge destination was predicted by a patient's pre-operative beliefs and their age, rather than self-reported physical function, helping to identify before TKA surgery the patients that are most likely to discharge to inpatient rehabilitation. The early identification of patients that are most likely to discharge to inpatient rehabilitation allows for methods to provide targeted post-operative care and optimize resources.

The ARISE tool was designed to capture a patient's socio-demographic and functional characteristics as well as include questions about their pre-surgical beliefs towards about inpatient rehabilitation. Our results showed that the ARISE questions that were highly predictive of inpatient rehabilitation were those that asked about a patient's beliefs about rehabilitation. This finding is in keeping with the results of other predictive studies that demonstrated a patient's "preferred discharge destination" was the most predictive variable of 
actual discharge destination $[14,16,17]$. However, the ARISE tool goes further, identifying potential reasons why a patient would prefer to discharge to inpatient rehabilitation.

There is existing research that older age is predictive of discharge destination after TKA [2429], which is consistent with our results suggesting that even when accounting for other predictive variables, age of 75 years or greater resulted in a greater likelihood of inpatient rehabilitation discharge. Prior reports of other demographic variables being predictive of discharge destination after TKA, including female gender, increased co-morbidity and obesity, was not replicated in this study [24, 25, 27, 28, 30, 31]. However, the ARISE cohort demonstrated homogeneity across comorbidity and obesity scales, thus, a sample of size of 100 may not have been large enough to be sensitive to differences in these characteristics between groups.

The ARISE tool has some similar features to the well validated EQ-5D-5L instrument, in that it is a "domain-based" questionnaire and designed for self-completion, the ARISE tool also utilises a 5-item response scale as does the EQ-5D-5L. However, where the EQ-5D-5L is a standardised instrument for measuring generic health status, the ARISE tool has been developed to also include a socio-demographic domain and to question a patient's beliefs around inpatient rehabilitation before they have had their surgery. Although the EQ-5D-5L was not the primary outcome of interest in this trial, the lower score of the EQ-VAS in the inpatient rehabilitation discharge group demonstrates that this group believed they were in "worse health" both before and after their surgery than those who discharged home. Another outcome assessed in the ARISE trial was length of stay, with those discharging to inpatient rehabilitation staying one day longer than the control group. This result is difficult to interpret as when awaiting discharge to inpatient rehabilitation often operational characteristics dictate length of stay, such as bed availability.

A limitation of this study is its generalizability to other patients at other institutions. All surgeries were performed by experienced arthroplasty surgeons at a single high-volume institution. An enhanced recovery pathway, that included early mobilization was also utilized. Also, the differences in reported rates of inpatient rehabilitation between the private and public sectors is noted [11, 12], with patient preference likely carrying greater weight in the private sector. Therefore, these results may not be transferrable when different regimes are used or in patients who do not undergo elective primary TKA. 
219 The results of the ARISE trial suggest that interventions to facilitate home discharge after uncomplicated primary TKR may be best aimed towards modifiable factors such as increasing access to home domiciliary services and addressing a patient's pre-surgical beliefs about the perceived challenges of completing their rehabilitation at home. Perioperative advancements, such as multimodal pain management, blood management and early mobilization protocols all contribute to a rapid recovery pathway which has been reported to lower hospital length of stay (LOS) or same-day surgery without adversely impacting postoperative complications or readmissions [32]. When these improvements in TKA management are combined with a simple home rehabilitation program, lengthy and costly inpatient rehabilitation may be able to be avoided in many circumstances.

\section{Conclusion}

Pre-operative patient beliefs regarding rehabilitation and future home social support are highly predictive of discharge destination after primary TKA, while the only demographic variable that is predictive is increasing age, and in particular, age 75 years and over. Selfreported pre-operative physical function is not predictive of discharge destination. Patient's psychosocial status requires much greater pre-operative examination to avoid unnecessary discharge to inpatient rehabilitation.

Funding

This research did not receive any specific grant from funding agencies in the public, commercial, or not-for-profit sectors.

The authors have no competing interests to declare. 
1. Kurtz, S.M., et al., Impact of the economic downturn on total joint replacement demand in the United States: updated projections to 2021. The Journal of bone and joint surgery. American volume, 2014. 96(8): p. 624.

2. Culliford, D., et al., Future projections of total hip and knee arthroplasty in the UK: results from the UK Clinical Practice Research Datalink. Osteoarthritis and cartilage, 2015. 23(4): p. 594-600.

3. Ackerman, I.N., et al., The projected burden of primary total knee and hip replacement for osteoarthritis in Australia to the year 2030. BMC Musculoskeletal Disorders, 2019. 20(1): p. 90.

4. Hooper, G., et al., Current trends and projections in the utilisation rates of hip and knee replacement in New Zealand from 2001 to 2026. The New Zealand medical journal, 2014. 127(1401): p. 82-93.

5. $\quad \mathrm{Ng}, \mathrm{M}$., et al., Global, regional, and national prevalence of overweight and obesity in children and adults during 1980-2013: a systematic analysis for the Global Burden of Disease Study 2013. The Lancet, 2014. 384(9945): p. 766-781.

6. Cross, M., et al., The global burden of hip and knee osteoarthritis: estimates from the Global Burden of Disease 2010 study. Annals of the Rheumatic Diseases, 2014. 73(7): p. 1323.

7. Flego, A., et al., Addressing obesity in the management of knee and hip osteoarthritis - weighing in from an economic perspective. BMC Musculoskeletal Disorders, 2016. 17(1): p. 233.

8. Kleinert, S. and R. Horton, Rethinking and reframing obesity. The Lancet, 2015. 385(9985): p. 2326-2328.

9. Ramos, N.L., et al., Correlation Between Physician Specific Discharge Costs, LOS, and 30-day Readmission Rates: An Analysis of 1,831 cases. The Journal of Arthroplasty, 2014. 29(9): p. 1717-1722.

10. Bozic, K.J., et al., Bundled Payments in Total Joint Arthroplasty: Targeting Opportunities for Quality Improvement and Cost Reduction. Clinical Orthopaedics and Related Research®, 2014. 472(1): p. 188-193. 
11. Buhagiar, M.A., et al., Effect of Inpatient Rehabilitation vs a Monitored Home-Based Program on Mobility in Patients With Total Knee Arthroplasty: The HIHO Randomized Clinical Trial. Jama, 2017. 317(10): p. 1037-1046.

12. Schilling, C., et al., Predictors of inpatient rehabilitation after total knee replacement: an analysis of private hospital claims data. Medical Journal of Australia, 2018. 209(5): p. 222-227.

13. Cizmic, Z., et al., The Risk Assessment and Prediction Tool Is Less Accurate in Extended Length of Stay Patients Following Total Joint Arthroplasty. The Journal of Arthroplasty, 2019. 34(3): p. 418-421.

14. Oldmeadow, L.B., H. McBurney, and V.J. Robertson, Predicting risk of extended inpatient rehabilitation after hip or knee arthroplasty. The Journal of Arthroplasty, 2003. 18(6): p. 775-779.

15. Sconza, C., et al., The Risk Assessment and Prediction Tool (RAPT) after Hip and Knee Replacement: A Systematic Review. Joints, 2019. 07(02): p. 041-045.

16. Konopka, J.F., et al., Risk assessment tools used to predict outcomes of total hip and total knee arthroplasty. The Orthopedic clinics of North America, 2015. 46(3): p. 351-x.

17. Halawi, M.J., et al., Patient Expectation Is the Most Important Predictor of Discharge Destination After Primary Total Joint Arthroplasty. The Journal of Arthroplasty, 2015. 30(4): p. 539-542.

18. von Elm, E., et al., The Strengthening the Reporting of Observational Studies in Epidemiology (STROBE) statement: guidelines for reporting observational studies. Journal of clinical epidemiology, 2008. 61(4): p. 344-349.

19. Artino, A.R., et al., Developing questionnaires for educational research: AMEE Guide No. 87. Medical Teacher, 2014. 36(6): p. 463-474.

20. Herdman, M., et al., Development and preliminary testing of the new five-level version of EQ-5D (EQ-5D-5L). Quality of life research : an international journal of quality of life aspects of treatment, care and rehabilitation, 2011. 20(10): p. 17271736.

21. Van Onsem, S., et al., A New Prediction Model for Patient Satisfaction After Total Knee Arthroplasty. The Journal of Arthroplasty, 2016. 31(12): p. 2660-2667.e1.

22. Brander, V.A., et al., Predicting total knee replacement pain: a prospective, observational study. Clinical orthopaedics and related research, 2003(416): p. 27-36. 
23. Sattler, L.N., W.A. Hing, and C.J. Vertullo, Pedaling-Based Protocol Superior to a 10-Exercise, Non-Pedaling Protocol for Postoperative Rehabilitation After Total Knee Replacement: A Randomized Controlled Trial. The Journal of bone and joint surgery. American volume, 2019. 101(8): p. 688-695.

24. Crawford, D.A., et al., Preoperative predictors of length of hospital stay and discharge disposition following primary total knee arthroplasty at a military medical center. Mil Med, 2011. 176(3): p. 304-7.

25. Murphy, B.P.D., et al., The impact of older age on patient outcomes following primary total knee arthroplasty. Bone Joint J, 2018. 100-b(11): p. 1463-1470.

26. Prohaska, M.G., et al., Preoperative body mass index and physical function are associated with length of stay and facility discharge after total knee arthroplasty. Knee, 2017. 24(3): p. 634-640.

27. Rissman, C.M., et al., Predictors of Facility Discharge, Range of Motion, and Patient-Reported Physical Function Improvement After Primary Total Knee Arthroplasty: A Prospective Cohort Analysis. J Arthroplasty, 2016. 31(1): p. 36-41.

28. Schwarzkopf, R., et al., Factors Influencing Discharge Destination After Total Knee Arthroplasty: A Database Analysis. Geriatr Orthop Surg Rehabil, 2016. 7(2): p. 95-9.

29. Sikora-Klak, J., et al., The Effect of Comorbidities on Discharge Disposition and Readmission for Total Joint Arthroplasty Patients. J Arthroplasty, 2017. 32(5): p. 1414-1417.

30. D'Apuzzo, M.R., W.M. Novicoff, and J.A. Browne, The John Insall Award: Morbid obesity independently impacts complications, mortality, and resource use after TKA. Clin Orthop Relat Res, 2015. 473(1): p. 57-63.

31. Sayeed, Z., et al., Comparing In-Hospital Total Joint Arthroplasty Outcomes and Resource Consumption Among Underweight and Morbidly Obese Patients. J Arthroplasty, 2016. 31(10): p. 2085-90.

32. Sibia, U.S., J.H. MacDonald, and P.J. King, Predictors of Hospital Length of Stay in an Enhanced Recovery After Surgery Program for Primary Total Hip Arthroplasty. The Journal of Arthroplasty, 2016. 31(10): p. 2119-2123. 
346 Figure 1. The ARISE (Arthroplasty Rehabilitation Initial Screening Evaluation) tool.

\begin{tabular}{|l|l|l|}
\hline Name: & $\begin{array}{l}\text { Today's } \\
\text { Date: }\end{array}$ & $\begin{array}{l}\text { Surgery } \\
\text { Date: }\end{array}$ \\
\hline Phone: & Height: & Weight: \\
\hline Date of Birth: & First Knee Joint Replacement? Yes or No \\
\hline
\end{tabular}

347

Which of the following statements best describes your current living situation?

Tick one situation only

Someone else lives with me, and I take care of myself

Someone else lives with me, and I need help taking care of myself

I live alone, and I take care of myself

I live alone, and I need help taking care of myself

348

\begin{tabular}{|c|c|c|c|c|}
\hline \multirow[b]{2}{*}{ Can you tell us about your current situation? } & \multicolumn{4}{|c|}{ Tick one response for each question only } \\
\hline & $\begin{array}{l}\text { All of the } \\
\text { time }\end{array}$ & $\begin{array}{c}\text { Most of the } \\
\text { time }\end{array}$ & $\begin{array}{c}\text { Some of the } \\
\text { time }\end{array}$ & Not at all \\
\hline \multicolumn{5}{|l|}{ I can walk without a walking stick or walker easily } \\
\hline \multicolumn{5}{|l|}{ I can do my shopping easily } \\
\hline \multicolumn{5}{|l|}{ I can bathe/dress myself easily } \\
\hline \multicolumn{5}{|l|}{ I can drive myself to appointments on my own } \\
\hline \multicolumn{5}{|l|}{ I am confident when I climb stairs } \\
\hline I have someone who can help me after surger & & & & \\
\hline
\end{tabular}

\begin{tabular}{|l|l|l|l|l|}
\hline \multirow{2}{*}{ How do you feel about the following statements? } & \multicolumn{3}{|c|}{ Tick one response for each question only } \\
\cline { 2 - 5 } & $\begin{array}{c}\text { All of the } \\
\text { time }\end{array}$ & $\begin{array}{c}\text { Most of the } \\
\text { time }\end{array}$ & $\begin{array}{c}\text { Some of the } \\
\text { time }\end{array}$ & Not at all \\
\hline I am anxious about my upcoming surgery & & & & \\
\hline I am afraid of falling over & & & & \\
\hline $\begin{array}{l}\text { I am worried about being a burden on my family or friends } \\
\text { during my recovery }\end{array}$ & & & & \\
\hline
\end{tabular}

349

How much do you agree/disagree with each of the following statements?

I would prefer a therapist to supervise my exercises

I would do best staying in a rehabilitation facility instead of doing my rehabilitation at home

I would have difficulties following a home-based exercise program after my surgery 
353 Table 1. Patient characteristics and discharge destination

\begin{tabular}{|lllr|}
\hline Characteristic & Home $(\boldsymbol{n}=\mathbf{8 2})$ & Rehab $(\boldsymbol{n}=\mathbf{1 8})$ & $\boldsymbol{P}$-value \\
\hline Age (yrs.), Mean (SD) & $68.5(7.8)$ & $73.0(9.5)$ & $\mathbf{0 . 0 3 6}$ \\
Age $>75(\mathrm{n}=29), n(\%)$ & $20(24.4)$ & $(9) 50.0$ & $\mathbf{0 . 0 3 0}$ \\
$\begin{array}{l}\text { Gender, } n(\%) \\
\quad \text { Male }(n=46)\end{array}$ & $39(47.6)$ & $7(38.9)$ & 0.500 \\
Female $(n=54)$ & $43(52.4)$ & $11(61.1)$ & 0.608 \\
BMI (kg/m²), Mean (SD) & $29.8(4.9)$ & $29.1(6.6)$ & 0.558 \\
ASA, Median (Range) & $2.0(1.0-3.0)$ & $2.0(2.0-3.0)$ & \\
& & & \\
\hline
\end{tabular}

Table 2. ARISE questions predictive of discharge to rehabilitation after univariable logistic regression analysis

\begin{tabular}{|c|c|c|c|c|}
\hline Question & $\begin{array}{c}\text { Home } \\
(n=82) \\
n(\%)\end{array}$ & $\begin{array}{c}\text { Rehab } \\
(n=18) \\
n(\%)\end{array}$ & $\begin{array}{l}\text { Odds Ratio } \\
(95 \% \mathrm{Cl})\end{array}$ & $P$-value \\
\hline $\begin{array}{l}\text { I have someone who can help me after surgery if } \\
\text { needed } \\
\text { Response: "Some of the time or Not at all" }\end{array}$ & $7(8.5)$ & $9(50)$ & $\begin{array}{c}10.7 \\
(3.2 \text { to } 35.8)\end{array}$ & $<0.001$ \\
\hline $\begin{array}{l}\text { I am worried about being a burden on my family or } \\
\text { friends during my recovery } \\
\text { Response: "Most or All of the time" }\end{array}$ & 11 (13.4) & $10(55.6)$ & $\begin{array}{c}8.1 \\
(2.6 \text { to } 24.9)\end{array}$ & $<0.001$ \\
\hline $\begin{array}{l}\text { I would prefer a therapist to supervise my exercises } \\
\text { Response: "Agree or Strongly agree" }\end{array}$ & $32(39.0)$ & $14(77.8)$ & $\begin{array}{l}5.5 \\
(1.7 \text { to } 18.1)\end{array}$ & 0.005 \\
\hline $\begin{array}{l}\text { I would do best staying in a rehabilitation facility } \\
\text { instead of doing my rehabilitation at home } \\
\text { Response: "Agree or Strongly agree" }\end{array}$ & $11(13.4)$ & $15(83.3)$ & $\begin{array}{c}32.8 \\
(8.0 \text { to } 129.9)\end{array}$ & $<0.001$ \\
\hline $\begin{array}{l}\text { I would have difficulties following a home-based } \\
\text { exercise program after my surgery } \\
\text { Response: "Agree or Strongly agree" }\end{array}$ & $6(7.3)$ & $6(33.3)$ & $\begin{array}{c}6.3 \\
(1.8 \text { to } 22.9)\end{array}$ & 0.005 \\
\hline int & & & & \\
\hline
\end{tabular}


Table 3. ARISE questions predictive of discharge to rehabilitation after multivariable regression analysis

\begin{tabular}{|l|c|c|}
\hline Variable & $\begin{array}{c}\text { Odds Ratio } \\
\text { (95\% Cl) }\end{array}$ & P-value \\
\hline Age $>75$ & 3.6 (0.9 to 13.6) & $\mathbf{0 . 0 3 0}$ \\
\hline $\begin{array}{l}\text { I have someone who can help me after surgery if needed } \\
\text { Response: "Some of the time or Not at all" }\end{array}$ & 6.3 (1.5 to 26.8) & $<0.001$ \\
\hline $\begin{array}{l}\text { I would prefer a therapist to supervise my exercises } \\
\text { Response: "Most or All of the time" }\end{array}$ & \\
$\begin{array}{l}\text { I would do best staying in a rehabilitation facility instead of doing my } \\
\text { rehabilitation at home } \\
\text { Response: "Agree or Strongly agree" }\end{array}$ & $* 9.9$ (2.6 to 37.9) \\
$\begin{array}{l}\text { I would have difficulties following a home-based exercise program after } \\
\text { my surgery } \\
\text { Response: "Agree or Strongly agree" }\end{array}$ & \\
$\begin{array}{l}\text { P-value <0.05 statistically significant } \\
\text { *When a patient "agreed" to at least } 2 \text { of the } 3 \text { statements }\end{array}$ & \\
\hline
\end{tabular}

361 Table 4. EQ-5D-5L and Length of Stay and discharge destination

\begin{tabular}{|l|c|c|c|}
\hline Variable & $\begin{array}{c}\text { Home }(\boldsymbol{n}=\mathbf{8 2}) \\
\text { Median (Range) }\end{array}$ & $\begin{array}{c}\text { Rehab }(\boldsymbol{n}=\mathbf{1 8}) \\
\text { Median (Range) }\end{array}$ & $P$-value \\
\hline $\begin{array}{l}\text { EQ-5D-5L Score } \\
\text { (Pre-operative) }\end{array}$ & $11(6-20)$ & $12(8-19)$ & 0.211 \\
\hline $\begin{array}{l}\text { EQ-5D-5L Score } \\
\text { (Post-operative) }\end{array}$ & $10(6-18)$ & $10(7-18)$ & 0.095 \\
\hline $\begin{array}{l}\text { VAS Score (100-point scale) } \\
\text { (Pre-operative) }\end{array}$ & $82.5(30-100)$ & $72.5(30-100)$ & $\mathbf{0 . 0 4 3}$ \\
\hline $\begin{array}{l}\text { VAS Score (100-point scale) } \\
\text { (Post-operative) }\end{array}$ & $80(30-100)$ & $70(45-95)$ & $\mathbf{0 . 0 0 9}$ \\
\hline $\begin{array}{l}\text { Length of stay } \\
\text { (Days) }\end{array}$ & $3.0(1-7)$ & $4.0(2-9)$ & $<.001$ \\
\hline$P$-value <0.05 statistically significant & & & \\
\hline
\end{tabular}

